Todos los encargos de traducción de piezas teatrales para ser representadas, tanto si se trata de clásicos como de textos actuales, comparten unos requisitos básicos para que el texto funcione sobre el escenario. El objetivo del presente artículo es ilustrar, con ejemplos extraidos de la propia experiencia del autor como traductor teatral, los aspectos textuales que requieren más atención y los diversos caminos explorables para conseguir que el texto llegue al espectador con la máxima inmediatez y fluidez, y para recrear con eficacia todo el potencial de efectos y reacciones que subyace en el original. Es, a su vez, una reivindicación de la invisibilidad del traductor como condición esencial para cumplir dichos requisitos.

PaLABRAS CLAVE: funcionalidad, oralidad, inteligibilidad, ritmo, eufonía, lenguaje poético, lenguaje coloquial, invisibilidad del traductor.

\title{
Funcional e invisible
}

Joan Sellent Arús

Universitat Autònoma de Barcelona

All translations commissioned by theatrical companies, whether they are classics or present-day plays, share some basic requirements for the text to work dramatically. This article attempts to illustrate, through a number of examples drawn from its author's own experience as a translator for the stage, the more relevant textual aspects and the variety of paths through which a translation can be made as accessible as possible to the audience while at the same time recreating the potential effects and feedbacks that underlie the source text. It is also a vindication of the translator's invisibility as an essential condition for the attainment of these aims.

KEYWORDS: functional, orality, intelligibility, rhythm, euphony, poetic language, colloquial language, translator's invisibility. 
Debo advertir de entrada que éste no es un artículo académico; sólo pretende ser un relato fragmentario - acompañado de una serie 84 de reflexiones y ejemplos ilustrativos- de mi experiencia como traductor de textos en lengua inglesa para la escena catalana, actividad a la que me he dedicado de forma preferente en los últimos diez años.

En otoño de 1998 recibí el encargo de traducir Hamlet al catalán para la compañía de Lluís Homar. Hasta entonces había trabajado en la traducción de narrativa y guiones cinematográficos y televisivos, y, en el ámbito teatral, había hecho media docena de traducciones en colaboración con Ferran Toutain. Hamlet fue mi primera versión teatral en solitario, y los criterios que en ella apliqué son los que han presidido todos los demás encargos de traducción para la escena que he realizado hasta la fecha.

Lluís Homar quería una versión que, sin rebajar excesivamente la carga retórica ni el registro poético del original, potenciara la inteligibilidad sirviéndose de unos materiales lingüísticos contemporáneos y utilizándolos de una forma tan llana y transparente como permitiera la complejidad del texto inglés, con los mínimos obstáculos que pudieran entorpecer la dicción de los actores y la recepción del mensaje verbal por parte del público. Una de las prioridades de aquel proyecto de puesta en escena era desmentir la creencia de que un clásico es algo indefectiblemente asociado a un discurso espeso y arcaizante, y a cuya plena comprensión sólo tienen acceso unos pocos iniciados.

Shakespeare siempre ha estado presente en la programación de los teatros catalanes. Durante muchos años, para las puestas en escena de este autor en catalán se recurría casi siempre a las traducciones del poeta y dramaturgo Josep Maria de Sagarra, unas versiones en verso endecasílabo de un vuelo poético y una musicalidad

innegables, reflejo del enorme talento del traductor para la versificación, de su robusto sentido de la lengua y de su dominio de todos los recursos expresivos del catalán. Sin embargo, en la jerarquía de prioridades de esas versiones no siempre ocupa un lugar preferente la inteligibilidad inmediata del texto: los enunciados están sujetos a una regularidad métrica inflexible, algo que a menudo comporta cierta violencia sintáctica y unas licencias léxicas y locucionales que combinan arcaísmos con coloquialismos, no siempre pertinentes al registro que impone el original.

Yo mismo - si se me permite la alusión directa a mi propia experiencia como espectador-, cuando era un joven aficionado al teatro y asistía a las representaciones de Shakespeare en catalán durante los años setenta y ochenta del siglo pasado, salía siempre con la sensación de haberme quedado a medias. Había disfrutado de unos versos de una gran sonoridad y de una innegable brillantez idiomática, pero esta capa formal (donde, al lado de las servitudes métricas, los rasgos estilísticos propios de Sagarra enmascaraban en exceso el estilo del autor) me impedía a menudo descifrar exactamente lo que me decían aquellos versos. Me había perdido demasiados matices de significado para que no me embargara cierto complejo de inferioridad: «Shakespeare no está a tu alcance», pensaba; «no estás entre los privilegiados que pueden asimilarlo plenamente». Pero al mismo tiempo ya intuía que Shakespeare podía ser traducido y transmitido de otra forma, y que quizás no todos los problemas de comprensión eran atribuibles a mi ignorancia.

Por eso, cuando años después recibí el encargo de traducir Hamlet con los requisitos que he apuntado más arriba, se dio la coincidencia perfecta entre lo que se me pedía como traductor y lo que yo mismo había echado en 
falta como espectador. Tuve la oportunidad de poner en práctica unos criterios que, basándose en un modelo de lengua libre de arcaísmos y connotaciones no deseadas y en unas pautas de versificación lo bastante flexibles como para que la métrica no fuera en detrimento de la sintaxis ${ }^{\mathrm{T}}$, pudieran derivar en un texto más inteligible que los que años antes había escuchado desde el patio de butacas ${ }^{2}$.

Después de aquella primera incursión en solitario en el teatro profesional, los encargos de traducción que he ido recibiendo han sido de una gran diversidad: junto a otros textos de Shakespeare o de algún otro autor isabelino, también he tenido ocasión de traducir un buen número de obras de dramaturgos rigurosamente contemporáneos, así como de autores de la primera mitad del siglo xx o finales del xIX.

En principio, la gran distancia temporal, cultural y estilística que existe entre una obra de Shakespeare y la de un autor joven del siglo XIx (el irlandés Conor McPherson, por poner un ejemplo) podría hacer pensar que uno y otro texto requieren unos objetivos y unas estrategias de traducción muy distintos, pero la experiencia me demuestra que en realidad no es así. Los requisitos básicos del encargo de traducción —inteligibilidad y fluidez de los enunciados; en una palabra: funcionalidad- permanecen inalterables.

I En realidad, estos criterios siguen muy de cerca los aplicados por Salvador Oliva en su traducción al catalán de la obra completa de Shakespeare. Las traducciones de Oliva presentan, además, una fidelidad al sentido y al registro mucho más constantes que las de Sagarra, y también en este aspecto me sirvieron de referente.

2 Potenciar la inteligibilidad de un texto de Shakespeare es un objetivo que tiene sus riesgos y requiere una serie de matices y estrategias concretas para sortearlos. Para un análisis más detallado de esas estrategias, ver Joan Sellent Arús, «Entendre Shakespeare», Quaderns. Revista de traducció, I4, Bellaterra, UAB, Departament de Traducció i d'Interpretació, 2007, pp. I7I-I82.
Una de las prioridades para que el discurso resulte fluido es el control del ritmo. En una pieza teatral en verso es donde se hace más evidente la presencia del ritmo como uno de sus rasgos discursivos más conspicuos, puesto que viene marcado por unas pautas métricas visibles y perfectamente definidas. En cambio, en una obra de teatro en prosa, y sobre todo si el lenguaje se enmarca en un registro coloquial que pretende recrear el habla más espontánea, se podría pensar que precisamente ese esfuerzo por buscar la naturalidad excluye cualquier tipo de esquema rítmico y acentual subyacente; sin embargo, raramente es así: el discurso dramático coloquial más aparentemente espontáneo suele ser el resultado de un trabajo minucioso en los niveles del ritmo, la eufonía y otros aspectos que contribuyen a que su transmisión resulte fluida y libre de estorbos; la transmisión del sentido y la ilusión de naturalidad están en función estricta del control de dichos elementos: las cacofonías, las ambigüedades semánticas no deseadas, las arritmias, las redundancias rítmicas o las rimas internas (algo que el hablante que se expresa espontáneamente, como es lógico, no está pendiente de controlar) son obstáculos que, sobre un escenario, van en detrimento de la credibilidad de la interpretación y de la clara transmisión del mensaje. Y una representación teatral nunca debería ser una carrera de obstáculos por culpa del texto.

Un fragmento cualquiera de Shining City, del ya citado Conor McPherson, puede servir para ilustrar la capacidad del autor para construir —sobre la base de un material «impuro» que se nutre de vacilaciones, síncopas, tics y latiguillos idiomáticos, leves incorrecciones sintácticas y groserías descarnadas - toda una poética de la lengua oral, con unas cadencias y una pulsación rítmica que tienen mucho más en común con 
un texto en verso de lo que pueda parecer a primera vista:

86

You see, we'd, I think, we'd been slightly left behind, a little bit, you know? All our... all our friends, they, you know, they had families. And, that... that... bound them together, you know? And, you see, I think that that... that we were... we were slightly left behind a little bit maybe. And that we felt that there was something kind of wrong with us, not anything serious or really wrong, but there was a whole... you know, a whole experience, a whole way, maybe, of... or relating to everything, that wasn't... it wasn't available to us. (Suddenly.) It was a pain in the fucking hole to tell you the truth! (Shining City, escena 3, p.3I)

Teniendo muy presente todo este aparato retórico y trasladándolo al marco de los mecanismos coloquiales de la lengua de llegada, así es como traduje este fragmento en la versión al catalán (Una ciutat brillant) que redacté por encargo del Centre d'Arts Escèniques de Terrassa:

Perquè diguéssim que ens havíem anat quedant com... marginats, una mica marginats, m'entens? Tots els... tots els nostres amics havien... en fi, que tots tenien fills, i... i això... i això els... els feia estar més units, saps què vull dir? I em sembla que... això que et deia, que una mica vam anar quedant... marginats, potser com una mica deixats de banda. I això feia que tinguéssim la sensació que entre nosaltres hi havia alguna cosa que... que no... que no acabava de funcionar, vull dir no era cap cosa greu ni allò que dius quin desastre, però era la sensació que hi havia tot un... tota una experiència concreta, tota una manera de... de connectar amb les coses, que nosaltres no... no hi teníem... accés. (Sobtadament.) I era una grandíssima tocada de collons, si vols que t'ho digui!

Es conveniente que el traductor ejerza siempre el mismo control minucioso que el autor al abordar todos los elementos formales en el texto de llegada. Esta minuciosidad requiere detenerse en aspectos textuales que, tomados de forma aislada, pueden parecer francamente menores (y probablemente lo son), pero cuya reiteración acaba incidiendo negativamente en la comodidad interpretativa de los actores y, por lo tanto, en la fluidez y la eufonía general del discurso. En mi versión catalana del monólogo I Am My Own Wife (Jo sóc la meva dona), de Doug Wright, ante la frase «he's a real dictator» rechacé la opción «és un autèntic dictador» por la cacofonía que deriva del contacto silábico «tic/dic» y barajé otras posibilidades, como «és un veritable dictador» $\mathrm{o}$ «es un autèntic dèspota», optando finalmente por la segunda.

Tomemos unos cuantos ejemplos de la obra de George Bernard Shaw Heartbreak House ( $\mathrm{La}$ casa dels cors trencats), que traduje por encargo del Teatre Nacional de Catalunya:

LADY UTTERWORD $\quad[\ldots]$ Randall: how dare you? RANDALL How dare I what?...

(Heartbreak House, acto I, p. 504)

En este caso, ante la disyuntiva de traducir la primera pregunta como «Randall, com t'atreveixes» o «Randall, com t'atreveixes a...?», me decidí por la segunda opción porque me pareció que la presencia de la preposición después del verbo ofrecía un mejor encaje rítmico con la réplica. En la misma obra aparece la secuencia:

MAZZINI No: I'm no good at making money. I don't care enough for it, somehow,

(Heartbreak House, acto II, p. 525)

que en una primera opción traduje como:

MAZZini No: jo no serveixo, per fer diners. Perquè és que no m'importen gaire, en realitat. 
Después, sin embargo, decidí someterla a un pequeño cambio de orden sintáctico:

MAzzini No: jo no serveixo, per fer diners. Perquè és que, en realitat, no m’importen gaire.

Al evitar la repetición del esquema «sintagma verbal + sintagma preposicional», la pauta entonativa es menos redundante y el segmento mejora rítmicamente.

Del orden sintáctico depende a veces que se produzcan o no pequeñas cacofonías derivadas del contacto entre fonemas idénticos a final y principio de palabra. En el segundo acto aparece el enunciado siguiente:

ELLIE $\quad[\ldots]$ We had a talk after dinner; and he fell asleep in the middle of it.

(Heartbreak House, acto II, p. 528)

En una primera versión escribí: «Havent sopat estàvem parlant, i a mitja conversa s'ha quedat adormit», pero luego modifiqué el orden de la segunda frase a favor de: «[...] i s'ha quedat adormit a mitja conversa», con lo cual evité el contacto «conversa / s'ha» (y, de paso, seguí más de cerca el orden sintáctico del original).

En un determinado momento del primer acto, un personaje dice: «[...] when I meet a man that makes a hundred thousand a year, I take off my hat to that man, and stretch out my hand to him and call him brother». En mi versión escribí: «[...] quan conec un home que guanya cent mil lliures a l'any, em trec el barret, el saludo cordialment i l'anomeno germà». Es decir, en el penúltimo predicado («stretch out my hand to him») evité la opción más literal «li ofereixo la mà» para evitar la rima interna entre «mà» y «germà».

Las rimas son especialmente molestas cuando aparecen al final de dos réplicas consecutivas:
MANGAN I ruined your father, didn't I?

ELLIE Oh, not intentionally.

MANGAN Yes I did. Ruined him on purpose.

Heartbreak House, acto II, pp. 517-518)

MANGAN Jo vaig arruïnar el seu pare, recordi-ho.

ELLIE No pas intencionadament.

MANGAN S'equivoca: vaig arruïnar-lo expressament.

La rima en -ment de esta primera opción me hizo buscar alternativas, con el resultado siguiente:

MANGAN Jo vaig arruïnar el seu pare, recordi-ho.

ELLIE No pas intencionadament.

MANGAN S'equivoca: va ser deliberat.

En un diálogo espontáneo, todos estos rasgos de la capa más externa del lenguaje (es decir, sus componentes sonoros) suelen pasar mucho más desapercibidos. Los participantes en el intercambio verbal no están pendientes de si en los enunciados aparecen aliteraciones, cacofonías, arritmias o rimas internas; lo que importa es que los mensajes lleguen de forma clara al interlocutor. Por poner un ejemplo: en el contexto de una conversación real, lo más probable es que no se perciba ninguna anomalía en el hecho de que un hablante diga: «em despertés després d'haver dormit durant divuit hores»; lo altamente improbable, en cambio, es que acto seguido dicho hablante crea necesario disculparse por haber producido una cadena de aliteraciones tan extrema, sencillamente porque ni él mismo ni su interlocutor la habrán detectado. Sin embargo, cuando esta secuencia aparece en una traducción teatral y es el resultado de un primer intento de trasladar al catalán «to wake me up after I had slept eighteen hours» (Heartbreak House, acto II, p. 523), es muy poco recomendable que el traductor la dé por buena 
sin ningún retoque. Por eso descarté esta opción inmediatamente después de haberla tecleado y, tras una serie de tentativas de formulación, me incliné por: «em despertés al cap de divuit hores seguides de dormir».

Todas estas aparentes minucias de la capa más externa del lenguaje redundan en la funcionalidad global del texto sobre el escenario, pero, naturalmente, no acaba aquí la exigencia de un control textual minucioso. Ya hemos dicho que, cuanto más fluido es el mensaje, mejor es su recepción por parte del público; pero, por muy fluido que sea el texto, su recepción no será completa si no se controla también la claridad al nivel semántico, la comprensión inmediata de los enunciados.

Los problemas semánticos cuya resolución exige más a menudo alejarse de la literalidad son los juegos de palabras y las referencias al contexto sociocultural, donde muchas veces es imposible capturar la función y el efecto del original ciñéndose a sus mismos componentes semánticos. En estos casos, si se trata de una traducción destinada a ser leída, el traductor siempre tiene la posibilidad de ser literal y servirse de la nota a pie de página con la consabida fórmula: «Juego de palabras intraducible. N. del T.», a la cual podrá añadir, si le parece oportuno, una explicación del funcionamiento de dicho juego en la lengua original. En una traducción teatral para ser representada, en cambio - y por razones obvias-, este recurso le está vedado al traductor, y, si quiere salvar la eficacia del juego de palabras (es decir, provocar una reacción más o menos equivalente a la del espectador del texto original), no tendrá más remedio que inventar.

En la primera escena del segundo acto de King Richard II, de William Shakespeare (que en el momento de escribir estas líneas estoy traduciendo al catalán), John of Gaunt, grave-

mente enfermo, conserva aún la reserva suficiente de ironía para hacer un juego de palabras explotando la coincidencia de su nombre con el adjetivo «gaunt»:

KING RICHARD [...] How is't with agèd Gaunt? GAUNT O, how that name befits my composition! Old Gaunt indeed, and gaunt in being old. Within the Grief hath kept a tedious fast, And who abstains from meat that is not gaunt?

For sleeping England long time have I watched;

Watching breeds leanness, leanness is all gaunt.

The pleasure that some fathers feed upon

Is my strict fast - I mean my children's looks,

And therein fasting hast thou made me gaunt.

Gaunt am I for the grave, gaunt as a grave,

Whose hollow womb inherits naught but bones.

KING RICHARD Can sick men play so nicely with their names?

(II.i.72-84)

Un pasaje de este tipo es de los que tan a menudo pueden desmoralizar al traductor ante un texto de Shakespeare, sobre todo si se aferra a la consigna del «todo o nada» y pretende capturar todos los matices del original. Pero esta pretensión es absolutamente improductiva, y la única opción realista es aspirar a salvar lo que se pueda.

La réplica del rey nos da una pauta muy clara sobre lo que hay que salvar a toda costa: no se puede sacrificar el juego de palabras, puesto que la pregunta que formula al final del fragmento no tendría ninguna coherencia discursiva. Algo deberá idear el traductor para justificar que el rey pregunte a John of Gaunt (Joan de Gant, en catalán): «¿Pot, un home malalt, fer jocs amb el seu nom?» 
Pero el problema principal - y lo que obliga irremisiblemente a alejarse de la literalidad- es que la lengua de llegada no posee el adjetivo «gant». Lo más parecido fonéticamente es el sustantivo «guant» y parece que por esta vía se puede llegar a alguna solución que cumpla los requisitos, aunque sólo los cumplirá parcialmente porque «guant» («guante», en español) no posee por sí solo la connotación directa de flaco o demacrado del adjetivo inglés. De todos modos, ésta es la opción que adopté, con el resultado siguiente:

$$
\text { RICARD [...] Què fa el vell Gant? }
$$

GANT Quin nom més adient, pel meu estat! «Vell Gant», sí, en efecte: vell i magre.

A dintre meu la pena ha fet un llarg dejuni, $i$ ¿qui pot dejunar sense aprimar-se?...

Ja fa molt temps que vetllo l'Anglaterra adormida,

$i$ les vetlles desgasten, $\mathrm{i}$ el desgast aprima.

El vell Gant l'han privat d'aquell plaer amb què altres pares s'alimenten: veure els fills,

i el vell Gant, dejunant, s'ha anat quedant com un guant envellit, que ja només és bo per enfundar un sac d'ossos... un «vell guant».

RICARD ¿Pot, un home malalt, fer jocs amb el seu nom?

Salta a la vista que mi versión se ciñe a la consigna de «salvar lo que se pueda», aunque no tengo claro que sea mucho lo que he sido capaz de salvar: el juego de palabras pierde reiteración e inmediatez, y requiere muletas explicativas. $\mathrm{E}$ juego "Gant» / "guant» no hace su entrada hasta los dos últimos versos, mientras que, en el original, «Gaunt» / "gaunt» ya aparece en el segundo. Hay un intento de que el segundo verso retenga una mínima coherencia con el primero, salvando sólo el componente semántico de «old» con «vell» (viejo, en español) y añadiéndole el adjetivo «magre» (delgado, flaco, en español) para que el sentido del adjetivo inglés «gaunt» no se pierda del todo. La licencia de colocar el sintagma «un vell guant» al final del último verso responde a un intento de potenciar la recepción del juego de palabras por parte del espectador. También creí oportuno incluir, en los versos antepenúltimo y penúltimo, la aliteración «Gant» / «dejunant»/ «quedant» / «guant»; me pareció una licencia justificable, en el sentido de que podía servir de pequeña compensación al balance de pérdidas al reforzar el perfil del personaje como aficionado al juego verbal.

Las referencias al contexto sociocultural más inmediato son habituales en cualquier pieza teatral, con independencia del género y la época a que pertenezca. Tomemos un ejemplo del primer acto de Lady Windermere's Fan, de Oscar Wilde:

LORD DARLINGTON It's a curious thing, Duchess, about the game of marriage - a game, by the way, that is going out of fashion-the wives hold all the honours, and invariably lose the odd trick.

Duchess of BERWICK The odd trick? Is that the husband, Lord Darlington?

LORD DARLINGTON It would be rather a good name for the modern husband.

\section{(Lady Windermere's Fan, acto I, p. II)}

Este fragmento de diálogo contiene un uso metafórico de una terminología especializada: ironiza sobre el matrimonio utilizando un símil alusivo al bridge, uno de los juegos de salón más arraigados en la sociedad británica. La terminología propia de este juego: "the honours», «the old trick», resulta lo bastante familiar a un espectador anglófono como para que el recurso humorístico surta su efecto inmediato; en cambio, difícilmente se conservaría dicho efecto en una traducción literal al catalán o al castellano.

En la versión catalana (El ventall de Lady 
Windermere), que preparé por encargo del Teatre Nacional de Catalunya, reelaboré el fragmento citado aprovechando parcialmente los elementos léxicos del original pero eliminando la referencia al bridge, e introduje otros elementos de cosecha propia con la intención prioritaria de obtener un efecto humorístico más o menos equivalente:

LORD DARLINGTON És ben curiós, duquessa, aquest joc del matrimoni... un joc, per cert, que ja comença a passar de moda... però és molt curiós, perquè la dona s'endú tots els honors $i$, en canvi, en el balanç final, el saldo sempre va en contra d'ella.

Duquessa dE BERWICK Quan diu «el saldo», ¿es refereix al marit, Lord Darlington?

LORD DARLINGTON Seria un nom bastant adequat per als marits d'avui dia.

E1 doble uso metafórico de la palabra «saldo», que en su segunda aparición ha desplazado el sentido de «resultado» al de "persona o cosa de poco valor», muy arraigado en el catalán coloquial, cumplió el requisito del efecto inmediato y el público reaccionó con una hilaridad bastante satisfactoria.

Otro fenómeno textual al que hay que dedicar especial atención, al traducir una pieza teatral para ser representada, es el de las ambigüedades semánticas. La mayoría de estas ambigüedades derivan de la homofonía, y el traductor no podrá detectarlas plenamente si no somete cada una de las palabras y frases a la prueba de la oralidad antes de darlas por buenas: es decir, sólo diciendo el texto en voz alta se asegurará de que no se le escapa ninguna ambigüedad o polisemia no deseada.

Al final de la tercera escena del primer acto de King Richard II aparece el verso: «Then England's ground, farewell! Sweet soil, adieu», en el que la coincidencia de los sinónimos

«ground» $\mathrm{y}$ «soil» plantea un grave problema para el traductor al catalán, una lengua en la que el equivalente literal del inglés "ground» y el castellano «suelo» es la palabra «sòl», que sólo se diferencia de «sol» por el acento diacrítico (es decir, son dos palabras fonéticamente idénticas); por lo tanto, su utilización en este contexto queda descartada por razones obvias. En mi versión sorteé el problema como pude, consciente de que se perdía el efecto de los sinónimos: «Adéu, doncs, terra anglesa! Dolça Anglaterra, Adéu...»

Al traducir al catalán Intimacy, la adaptación teatral de la novela de Hanif Kureishi, se me escapó una de estas ambigüedades al dar por buena la frase «Amb humitat», que en la fonética del catalán central suena prácticamente igual que «Han vomitat». En el transcurso de los ensayos, como era de esperar, el director y los actores detectaron el problema, pero en vez de buscar —o pedirme a mí que buscara- una frase alternativa, prefirieron sacar partido de la ambigüedad y añadieron un par de réplicas al texto:

XAVIER Amb humitat.

víctor Han vomitat? Qui ha vomitat?

Xavier No: humitat; que dic que hi ha humitat.

Y este pequeño añadido sumó un efecto humorístico a la escena. Las morcillas suelen ser peligrosas, pero en este caso creo que se actuó con buen criterio: la escena en la que aparecía se enmarcaba en un registro humorístico general, y de este modo se acentuó discretamente su comicidad sin incurrir en un grave pecado de traición.

A veces, las ambigüedades semánticas ya aparecen en el texto original y son deliberadas. Sirva como botón de muestra uno de los casos más ilustres de la historia del teatro: en el tercer 
acto de Hamlet, Shakespeare pone en boca del protagonista el sintagma «country matters» en el contexto de una conversación con Ofelia:

HAMLET Lady, shall I lie in your lap?

opHELIA No, my lord.

HAMLET I mean, my head upon your lap?

opHELIA Ay, my lord.

HAMLET Do you think I meant country matters?

(III.ii.IO7-III)

La mayoría de los especialistas coinciden en señalar que este "country matters» busca un doble sentido aprovechando la identidad fonética entre las cinco primeras letras de la palabra "country» y una de las «four-letter words» más obscenas de la lengua inglesa. En su momento estuve tentado de recurrir al verbo «encunyar» (acuñar en español), que en catalán central coincide fonéticamente con «enconyar» (encoñar, en español), y producir algo así como: «Penses que volia encunyar una frase nova?», pero la prudencia me aconsejó desestimar la opción y me conformé con: «Penses que em referia a coses baixes?» No sé lo que haría si tuviera que traducir Hamlet actualmente, pero debo confesar que a menudo me arrepiento de no haber sucumbido a aquella tentación ${ }^{3}$.

El esfuerzo que implica la recreación de todos estos juegos textuales se ve recompensado si el traductor, desde su invisibilidad (ya sea en

3 La utilidad de comprobar el efecto sonoro de cada palabra y cada frase para detectar ambigüedades es aplicable, a mi entender, a todo tipo de textos, no sólo a los que van directamente destinados a la transmisión oral. Mientras redactaba este artículo releí el libro de Octavio Paz Traducción: literatura y literalidad, y, en la página 77, tropecé con la frase: « «El músico de Saint-Merry» es uno de los poemas más turbadores y misteriosos de Apollinaire» ( $\mathrm{Paz}$, I990; la negrilla es mía). Ignoro si Octavio Paz contempló la posibilidad de que algún día se le ocurriera a alguien leer su texto en voz alta, pero me atrevería a decir que no. el patio de butacas o entre bastidores), percibe que la reacción del público responde más o menos al efecto deseado, pero sería absurdo esperar que dicha reacción fuera acompañada de un reconocimiento consciente, por parte del público, de las estrategias de traducción que hay detrás de cada uno de esos efectos. El espectador no tiene por qué notar la mano del intermediario en el texto que le llega, del mismo modo que no tiene por qué percibir conscientemente la mano del director en la interpretación y el movimiento de los actores.

En los escenarios, detrás de la verosimilitud interpretativa hay un trabajo invisible de dirección actoral y dramatúrgica. Asimismo, la naturalidad y la credibilidad del discurso son directamente proporcionales a la invisibilidad del traductor. Sin conocer a fondo las últimas corrientes de la traductología, me consta - si no he leído mal- que goza de un considerable predicamento la tendencia, abanderada por Lawrence Venuti (I995), a considerar la invisibilidad del traductor como algo seriamente cuestionable, si no directamente negativo. Pues bien: mucho me temo que, a un militante de la visibilidad del traductor, poco futuro le espera en los escenarios actuales. En las versiones del ya citado Josep Maria de Sagarra, su visibilidad es innegable y rotunda: ante una de sus traducciones de Shakespeare, a ratos nos da la impresión de que leemos o escuchamos una más de las obras de su repertorio propio; y algo tendrá que ver con ello el imparable retroceso de las puestas en escena de versiones sagarrianas.

La invisibilidad del traductor es claramente denunciable, por supuesto, si en el programa de un montaje teatral no aparece su nombre; constituye una práctica fraudulenta, e incluso impugnable por la vía legal. También la ausencia de cualquier mención 
al traductor en no pocas reseñas de estrenos teatrales es difícil de justificar, a menos que - puestos a ser optimistas - la atribuyamos al hecho de que el texto sonaba tan fluido, natural y creíble que el crítico no cayó en la cuenta de que la obra que había presenciado era una traducción ${ }^{4}$.

Pero, en lo que respecta al texto que se dice en escena, el trabajo del traductor debe pasar lo más desapercibido posible. Muy pocas frases del inglés admiten una única traducción al catalán, lo que significa que el traductor debe llevar a cabo un ejercicio constante de elección y descarte; la mejor prueba de que ha salido airoso de este ejercicio es que el espectador se lleve la impresión de que las frases que ha oído desde su butaca no podrían haber sido dichas de otra manera. Pero esto no hace más que corroborar la invisibilidad de quien ha hecho la traducción:

4 En la Guía del Ocio de Barcelona (semana del 3 al 9 de octubre de 2008) aparece la crítica al montaje de la obra Rock 'n' Roll, de Tom Stoppard, que traduje al catalán por encargo del Teatre Lliure y acababa de estrenarse, conservando el título original, bajo la dirección de Àlex Rigola. Cito textualmente algunos fragmentos: «... cabe reconocer a Rigola - y a su elenco — la capacidad de hacer digeribles unos diálogos densos, complicados, que exigen la máxima atención por parte del espectador (...) Entre todos, autor, director e intérpretes - más escenógrafo (Max Glaenzel) y figurinista (María Araujo) - logran sumergirnos en la Historia, hacernos partícipes de ella. Nos imparten una clase magistral que nadie debería perderse». Vaya por delante mi adhesión a los elogios que se dedican a todos estos profesionales: Rock 'n' Roll es uno de los montajes más redondos que se han visto últimamente en los escenarios catalanes. Al crítico, sin embargo, no se le ocurrió que la figura del traductor pudiera haber tenido alguna incidencia en el resultado global y en las virtudes que señala. No aparece por ninguna parte una sola referencia a la traducción ni al nombre del traductor, con lo cual no es descartable que algún lector poco informado, basándose en el dato estricto del título de la obra, llegara a pensar que Rigola y su elenco habían decidido representar la pieza de Stoppard en inglés. $¿ \mathrm{O}$ es que quizás el crítico en cuestión pensó que Tom Stoppard era tan buen conocedor del catalán que había escrito la obra directamente en esta lengua? Todo es posible, visto el elevado índice de desinformación de algunos críticos. sería absurdo esperar que el espectador se dijera cada dos por tres: «iQué bien ha resuelto las frases el traductor! Si es que ni parece una traducción...»

Pocos se han pronunciado tan radicalmente en este sentido como el poeta y traductor Josep Maria Fulquet, en un texto elaborado para el programa de mano de su versión de L’home, la bèstia $i$ la virtut, de Pirandello, estrenada en el Teatre Alegria de Terrassa en marzo de 2008. Es por ello que me permito transcribir sus palabras finales, pues creo que no hay mejor remate para lo que yo mismo he intentado argumentar: «Espero, doncs, que l'espectador surti de la sala sense saber en quina llengua li han parlat els actors; és a dir, que oblidi la figura de l'intermediari, del traductor, i s'identifiqui amb la paraula de l'autor».

Que una traducción teatral para ser representada resulte funcional depende del control de todos los aspectos textuales que he procurado ilustrar con ejemplos extraídos de mi propia experiencia. Si el traductor ha ejercido este control de forma competente, los responsables de defender el texto sobre el escenario se lo agradecerán. Para el director y los actores, la figura del traductor no suele ser invisible: ellos saben la diferencia entre un texto que potencia su trabajo y un texto que lo entorpece. Pocas cosas hay tan estimulantes para un traductor como oírles decir a los actores que su texto les ha resultado especialmente fácil de memorizar, y que se sienten cómodos al decir sus frases: ésta es la mejor garantía de que el requisito de funcionalidad se ha cumplido; es, en definitiva, la prueba inequívoca de que la mano invisible del traductor ha funcionado.

RECIBIDO DICEMBRE 2008 ACEPTADO FEBRERO 2009 


\section{REFERENCIAS BIBLIOGRÁFICAS}

Kureishi, H. (1999). Intimacy, Londres: Faber and Faber.

McPherson, C. (2005). Shining City, Londres: Nick Hern Books.

Paz, O. (1990). Traducción: literatura y literalidad, Barcelona: Tusquets Editores.

Sellent Arús, J. (2007). «Entendre Shakespeare». Quaderns. Revista de traducció, I4, Bellaterra: UAB, Departament de Traducció i d'Interpretació, pp. I7I-I82.

Shakespeare, W. (2005). King Richard II, C. R. Forker (ed.), Londres: Thomson Learning.
Shakespeare, W. (1982). Hamlet, H. Jenkins (ed.), Londres: Methuen.

Shaw, G. B. (1986). Heartbreak House, en S. Weintraub (ed.) The Portable Bernard Shaw, Harmondsworth: Penguin Books, pp. 44I-577.

Venuti, L. (1995). The Translator's Invisibility: A History of Translation, Londres: Routledge.

Wilde, O. (2000). Lady Windermere's Fan, en R. A. Cave (ed.) The Importance of Being Earnest and Other Plays, Londres: Penguin Books, pp. I-64.

Wright, D. (2005). I Am My Own Wife, Nueva York: Dramatists Play Service, Inc. 